\title{
Fabrication of a Maxillary Implant Retained Overdenture Using an Existing Subperiostal Implant: A Clinical Report
}

\author{
Carlos Barrero $^{1, *}$, Michael B. Border ${ }^{2}$ and Sompop Bencharit ${ }^{3}$ \\ ${ }^{I}$ Department of Prosthodontics, School of Dentistry, University of North Carolina, Chapel Hill, NC, USA \\ ${ }^{2}$ University of Mount Union, Alliance, OH, USA; and Research Assistant, Department of Prosthodontics, School of Den- \\ tistry, University of North Carolina, Chapel Hill, NC, USA \\ ${ }^{3}$ Department of Prosthodontics, School of Dentistry, and Department of Pharmacology, School of Medicine, University \\ of North Carolina, Chapel Hill, NC, USA
}

\begin{abstract}
Subperiosteal implants used to be prescribed to partially and fully edentulous patients to restore occlusion and esthetics prior to the emergence of the more successful endosseous implants that are used today. Because subperiosteal implants had a high incidence of failure, difficulty of placement, and post-operative complications, the use of subperiosteal implants declined significantly. However, some subperiostal implants placed 20-30 years ago still survive. Little information is available in the literature on how to treat patients whose subperiosteal implants still remain. This clinical case report thereby describes a treatment for a patient with a maxillary subperiosteal implant placed 23 years ago. The patient was offered a treatment option that included surgical implant removal, bone grafting and placement of endosseous implants to support a new maxillary overdenture. This treatment plan was not feasible due to the financial constraints of the patient and the complexity of the treatment. The patient chose a more conservative treatment plan, preserving the existing implant. The existing maxillary subperiosteal implant was restored with MICRO ERA attachments and a maxillary implant-retained overdenture was fabricated. The patient was satisfied with the esthetics and functional aspects of the treatment. No further peri-implant bone loss or other complications were found after a six-month recall. This clinical report suggests an alternative treatment plan for patients with existing subperiosteal implants that wish to avoid complex surgical procedures.
\end{abstract}

Keywords: Subperiosteal implant, overdenture, implant-retained overdenture, overdenture attachment.

\section{INTRODUCTION}

A subperiosteal implant refers to a type of dental implant that is placed in between the periosteum and the alveolar bone. It usually has two to four transmucosal elements projecting through the mucosa into the oral cavity, connecting the implant to the prosthesis. Traditionally, subperiosteal implants are made from chrome-cobalt or titanium alloys $[1,2]$ and often immediately loaded with a removable or fixed prosthesis similar to a system used for blade implants. They are usually placed above the bone and used in cases of severe bone resorption of the ridges [3, 4].

Some subperiosteal implants have shown poor biocompatibility resulting in inflammatory responses and rejection when alloys such as chrome-cobalt were used. However, the utilization of titanium greatly improved biocompatibility and improved rejection issues common with chrome cobalt alloys [5]. Corrosion resulting from the instability of this oxide-metal interface caused a foreign body reaction leading to chronic inflammation and eventually implant rejection and

*Address correspondence to this author at the Department of Prosthodontics School of Dentistry University of North Carolina, Chapel Hill, NC 27599-7450, USA; Tel: 919966 2712; Fax: 919966 3812;

E-mail: Carlos_Barrero@dentistry.unc.edu failure [6]. Secondly, a lack of osseointegration often results due to the nature of the surgical techniques and loading protocol traditionally used for subperiosteal implants. Conventionally, these chrome-cobalt subperiosteal implants would be placed on top of the bone and loaded with prostheses either immediately or soon after. Lack of direct bone contact and an immediate loading protocol often leads to fibrous integration [7]. Finally, treatments for failing implants are often complicated and require multiple surgeries and complex bone grafting procedures [8]. The popularity of subperiosteal implants greatly diminished after the introduction of the root-formed endosseous implants by PI Branemark in the early 80 's [9].

While the use of subperiosteal implants is limited today, there are still a small number of patients who survive with stable subperiosteal implants. Often these patients are presented with an option of implant removal, followed by grafting and placement of endosseous implants prior to the fabrication of a definitive prosthesis [10]. However, most of these patients are elderly who may have general health problems or may simply not want to pursue this option due to the complexity of the treatment and financial concerns. This case report presents a conservative approach for a patient who presented with a failing prosthesis that was retained by a stable maxillary subperiosteal implant. 


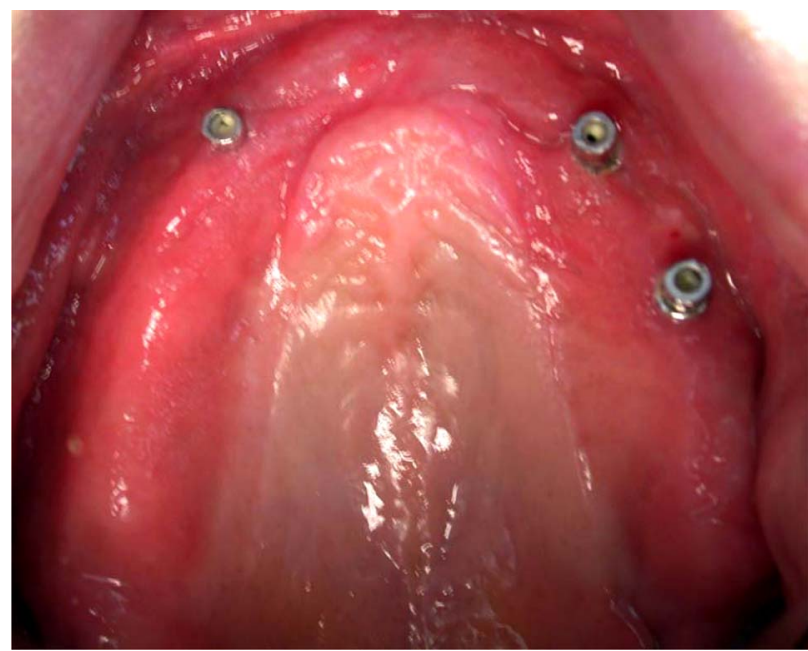

Fig. (1). Maxillary Arch.

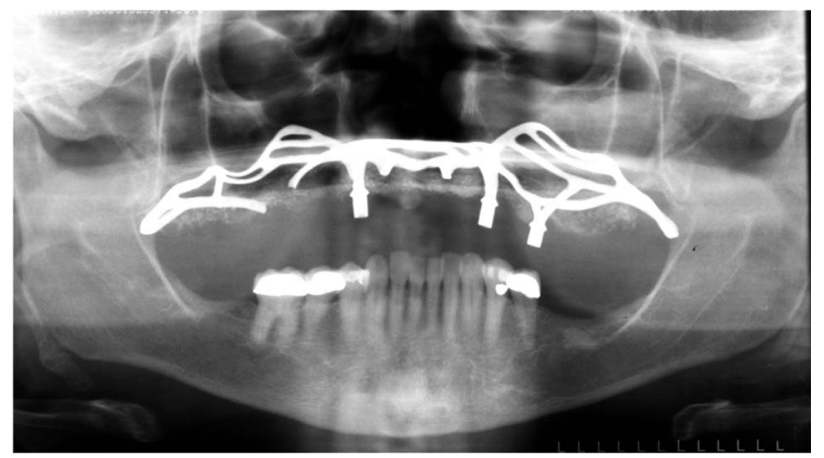

Fig. (2). Panoramic radiograph.

\section{CASE REPORT}

A 72-year-old African-American female presented to the Dental Faculty Practice at the University of North Carolina at Chapel Hill, School of Dentistry, with a chief complaint "My implant-denture is loose and the clips have broken, and I don't like how they feel anymore". The medical and dental history were reviewed and discussed with the patient. She presented with a history of cholecystectomy and thyrodectomy. She also presented with controlled type II diabetes, hypertension and hypercholesterolemia. Her vital signs were recorded on average as follows: blood pressure 135/86 mm $\mathrm{Hg}$, pulse 62 beats per minute, respiration 15 breaths per minute. She was a nonsmoker, and consumed alcohol occasionally (once a week). In 1986, a maxillary subperiosteal implant was placed and restored with a maxillary implant bar-retained overdenture. She had a mandibular removable partial denture (RPD) made at the same time to restore her posterior occlusion. The bars that retained the maxillary denture had recently fractured and the maxillary overdenture was no longer serviceable. In addition, the patient had recently had an episode of an acute infection in the upper right canine area. The infection was treated successfully by antibiotics, local scaling, and irrigation. A transmucosal element in the upper right molar area had been sectioned and removed because it had perforated the buccal mucosa creating an undercut that would not allow the maxillary denture to seat properly.

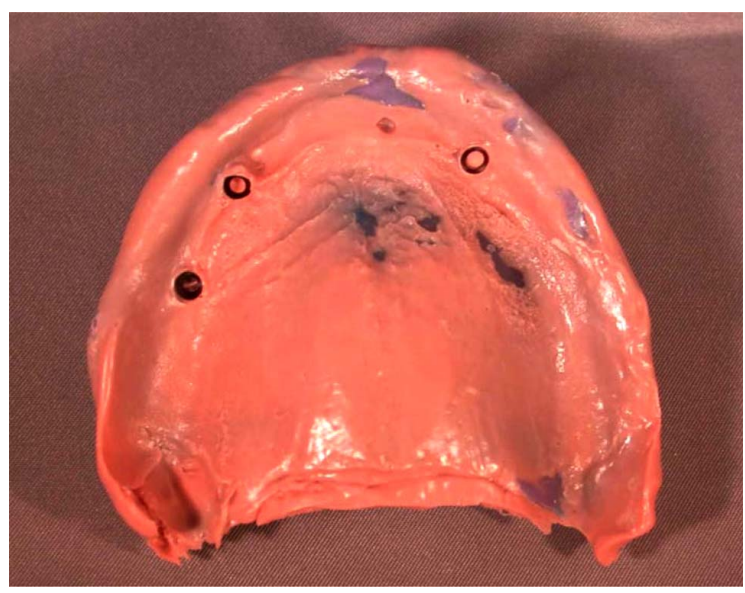

Fig. (3). Maxillary final impression.

Intra-oral examination showed that the maxillary overdenture was retained by two bar clip attachments that had debonded and were no longer retentive. However, the subperiosteal implant appeared to be stable with no signs of infection. The maxillary complete overdenture had little retention and stability. Generalized inflammation of tissue underneath the overdenture corresponding to type III Newton classification of denture stomatitis was also found. The mandibular arch, partially edentulous, was restored with a conventional Kennedy Class I mandibular RPD (Fig. 1).

A Panoramic image was taken to assess the condition of the maxillary and mandibular bones (Fig. 2) revealing normal mandibular bony architecture with moderate bone resorption loss in the posterior edentulous area. The maxilla showed a subperiosteal implant sitting over the remaining maxilla which had a substantial generalized area grafted with what appeared to be hydroxyapatite. There was a discontinuity of the implant section in the right molar region.

\section{TREATMENT}

A treatment option was presented to the patient including removal of the subperiosteal implant, bone grafting, and placement of endosseous implants, and fabrication of a new implant-retained overdenture or a new maxillary implantsupported fixed detachable prosthesis. This option was declined by the patient due to financial limitations and complexity of the treatment proposed. The second option was to utilize the existing subperiosteal implant to retain a new maxillary overdenture. This plan would include fabricating three custom overdenture Micro ERA attachments to the existing permucosal extensions of the implant, and fabricating a new maxillary implant-retained overdenture. The mandibular arch was not treated because it was determined that the partial denture had acceptable esthetic and functional components.

Preliminary maxillary and mandibular impressions were made with irreversible hydrocolloid (Jeltrate Plus, DENTSPLY $221 \mathrm{~W}$. Philadelphia, PA) and a stock tray to fabricate a maxillary custom tray with light cured acrylic resin (Triad VLC, DENTSPLY Philadelphia, PA) (Fig. 3). The maxillary overdenture was then relined with tissue conditioner (Coecomfort, GC America, Alsip, IL). The tissue conditioner was changed four times until the tissue under- 


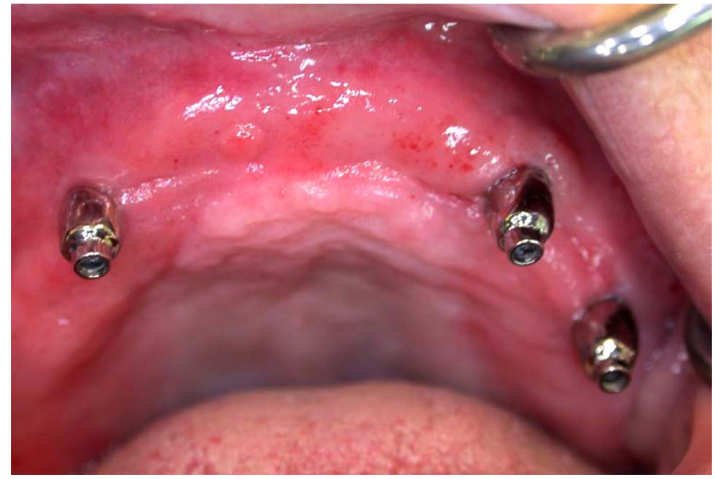

Fig. (4). Micro ERA cemented on subperiostal implant.

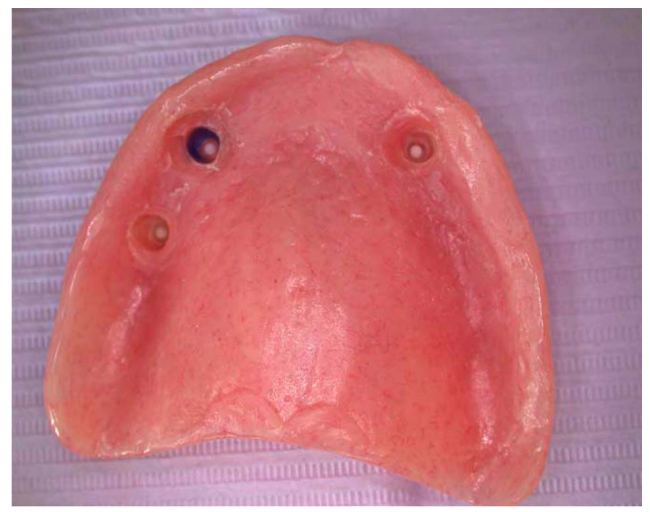

Fig. (5). Denture intaglio surface.

neath the denture appeared to return to the healthy state. The maxillary final impression was then made with a polyvinyl siloxane medium viscosity (Examix NDS, GC America, Alsip, IL) using a custom tray after a conventional border molding procedure with heavy body (Examix NDS, GC America, Alsip, IL). No modifications were made to the transmucosal elements of the implant. The form of these transmucosal elements provided sufficient retention and resistance to hold the MICRO ERA attachments in place. The tray was then removed from the mouth, and excess impression material was removed. The impression was evaluated for accuracy. The Impressions were poured with type III stone (Microstone. Whip Mix. 361 Louisville, KY). A mandibular impression with the removable partial denture in place was used to create an opposite cast. Finally, a maxillary cast was made to fabricate occlusal rims for a wax try in appointment (Fig. 4).

After obtaining maxillomandibular records with record bases and occlusion rims, the casts were transferred to a semi-adjustable Hanau ${ }^{\mathrm{TM}}$ articulator $96 \mathrm{H} 2$ (Whip Mix. Louisville, KY) using a face-bow transfer. Maxillary denture teeth (SR Ortholingual DCL, Ivoclar, Amherst, N.Y.) were selected and arranged on the record bases for a trial denture arrangement. The trial arrangement was evaluated intraorally for esthetics, phonetics, occlusal vertical dimension, and centric relation. A protrusive record was made to set the articulator's condylar elements, and a balanced occlusal arrangement was achieved. The patient's approval was obtained. The maxillary denture was processed and finished using conventional techniques.

Metal copings for sites 6, 11 and 13 were waxed and cast with a noble alloy (Argebond 80. Glastonbury, CT) using

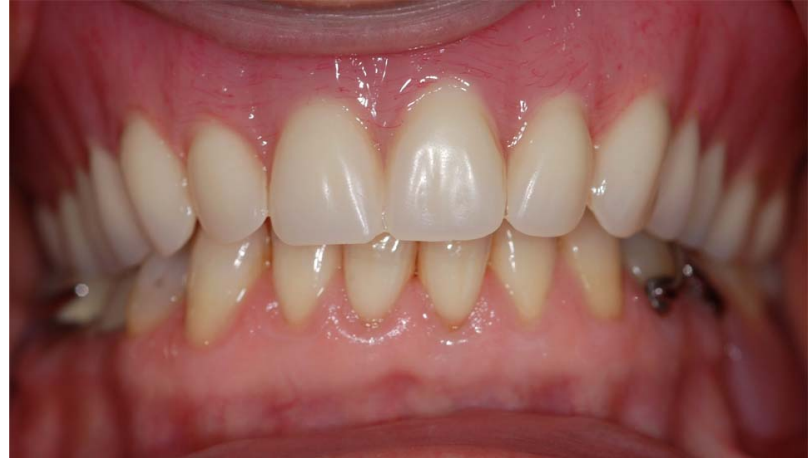

Fig. (6). Final prosthesis.

MICRO ERA cast-to patterns (Sterngold Dental, Attleboro, MA) to create custom overdenture abutments for sites 6,11 , and 13. Parallelism was achieved on the master cast by using a dental surveyor. Metal copings were intraorally evaluated, fitted and cemented with resin modified glass ionomer cement (Rely X Luting Plus Cement, 3M ESPE, St. Paul, MN). Before insertion of the maxillary denture, attachment sites in the intaglio surface were relieved. Patient approval was obtained. The maxillary denture was delivered. The patient was asked to return 24 hours and one week for post operatory appointments (Figs. 5 and $\mathbf{6}$ ).

The patient returned after one month to have the attachments processed into the denture using a "pick up" impression technique. Black processing attachments with housings were placed on the MICRO ERA attachments. GC hard relined material (GC America, Alsip, IL) was mixed and added to the relieved areas $(6,11$ and 13$)$ of the denture. The denture was seated in the patient's mouth. Upon setting of the material, the denture was removed and white attachments were replaced. Retention and stability was checked and approved by the patient.

\section{PROGNOSIS}

The patient was satisfied with the retention and stability of the maxillary overdenture. She was pleased with the functional and esthetic results of the treatment. The patient was placed on a six-month hygiene recall. This case report suggests that stable subperiosteal implants can be restored with MICRO ERA attachments and an implant-retained overdenture. This conservative treatment option should be considered as a viable alternative when the existing subperiosteal implants is stable with no signs and symptoms of a failing implant; and the removal of subperiosteal implants, followed by bone grafting and placement of endosteal implants is not accepted by the patient.

\section{ACKNOWLEDGEMENT}

The authors thank members of the University of North Carolina Department of Prosthodontics and the Dental Faculty Practice.

\section{CONFLICTS OF INTEREST STATEMENT}

The authors declare no conflict of interest.

\section{REFERENCES}

[1] Babbush C. Dental implants: the art and science. Philadelphia PA: Saunders, 2001; p. 476. 
[2] Balkin BE. Implant dentistry: historical overview with current perspective. J Dent Educ 1988; 52: 683-5.

[3] Linkow LI, Mangini F. Implant dentistry today: a multidisciplinary approach. Color Atlas Implant Tech prostheses. 1990; 5: 604.

[4] Alberto PL. Implant reconstruction of the jaws and craniofacial skeleton. Mt Sinai J Med 1998; 65: 316-21.

[5] Cantatore JL, Klein PA, Lieblich LM. Coetaneous dental sinus tract, a common misdiagnosis: a case report and review of the literature. Cutis 2002; 70: 264-7.

[6] Markiewicz MR, Nishiyama K, Yago K,et al. Draining orocutaneous fistula associated with a failing subperiosteal implant: report of a case. J Oral Implantol 2007; 33: 347-52.
[7] Esposito M, Thomsen P, Erickson LE, Sennerby L, Lekholm U. Histopathologic observations on late oral implant failures. Clin Implant Dent Relat Res 2000; 2: 18-32.

[8] Cutilli BJ, Smith BM, Bleiler R. Reconstruction of a severely atrophic maxilla using a Le Fort I downgraft and dental implants: clinical report. Implant Dent 1997; 6: 105-8.

[9] Adell R, Lekholm U, Rockler B, Branemark Pl. A 15-year study of osseointegrated implants in the treatment of the edentulous jaw. Int J Oral Surg 1981; 10: 387-416.

[10] Schou S, Pallesen L, Hjørting-Hansen E, Pedersen CS, Fibaek B. A 41-year history of a mandibular subperiosteal implant. Clin Oral Implants Res 2000; 11: 171-8.

(C) Barrero et al.; Licensee Bentham Open.

This is an open access article licensed under the terms of the Creative Commons Attribution Non-Commercial License (http://creativecommons.org/licenses/by-nc/3.0/) which permits unrestricted, non-commercial use, distribution and reproduction in any medium, provided the work is properly cited. 